\title{
Reliability and Validity of the Chinese Version of the Survey of Family Environment (SFE)
}

\author{
Junko Honda', Naohiro Hohashi ${ }^{1}{ }^{*}$, Sharron Kam Leung ${ }^{2}$ \\ ${ }^{1}$ Division of Family Health Care Nursing, Graduate School of Health Sciences, Kobe University, Kobe, Japan \\ ${ }^{2}$ School of Nursing, Hong Kong Baptist Hospital, Kowloon, Hong Kong, China \\ Email: *naohiro@hohashi.org
}

How to cite this paper: Honda, J., Hohashi, N. and Leung, S.K. (2016) Reliability and Validity of the Chinese Version of the Survey of Family Environment (SFE). Open Journal of Nursing, 6, 820-832. http://dx.doi.org/10.4236/ojn.2016.69081

Received: August 24, 2016

Accepted: September 26, 2016

Published: September 29, 2016

Copyright $\odot 2016$ by authors and Scientific Research Publishing Inc. This work is licensed under the Creative Commons Attribution International License (CC BY 4.0).

http://creativecommons.org/licenses/by/4.0/

\begin{abstract}
This study, in which 519 child-rearing families in Hong Kong participated, examined the reliability and validity of Chinese-language version of the Survey of Family Environment (SFE-C). The SFE-C is a self-administered questionnaire containing 30 items that examines family functioning and family support needs. It is designed to yield an instrument satisfaction score (SS score: family functioning score). Internal consistency was estimated at 0.92 (Cronbach's alpha for SS scores). In a test-retest study of 33 families, the correlation coefficient for families' mean SS score over a two-week period was 0.93 , indicating high test-retest reliability. Confirmatory and exploratory factor analysis using the Concentric Sphere Family Environment Theory indicated that the SFE-C's structure included seven factors, thereby supporting the SFE-C's construct validity. The SFE-C demonstrates good reliability and validity and may be used to evaluate Chinese families' functioning.
\end{abstract}

\section{Keywords}

Survey of Family Environment (SFE), Chinese, Instrument Development, Family Functioning, Concentric Sphere Family Environment Theory (CSFET)

\section{Introduction}

Increasing demand for family health care nursing has led nursing practitioners and researchers to focus on the family as a unit. Preparing undergraduate nurses to think in terms of the "family" crucially establishes family health care nursing as a core component of clinical practice [1]. In the past 20 years, researchers and practitioners have developed the family nursing theory/model and conducted family nursing research worldwide.

Several studies have examined family functioning in the Chinese population. The 
authors of the present research searched the PubMed database using variation of the terms "family functioning" and "Chinese" (searched on April 25, 2015). The search found that 86 papers were published between 1990 and 2014, of which over half were published after 2011, suggesting that academic interest in Chinese family functioning has increased over recent years. Some nursing research examined functioning in families that included patients with chronic conditions, such as diabetes or psychiatric illnesses. This research tended to address family functioning issues in terms of the whole family, rather than individual family members. Research that developed family functioning scales mainly took place in the 1970s and 1980s. Some family functioning scales were translated into Chinese, e.g., [2]-[6]. Although using the same scales between cultures offers certain advantages, e.g., it permits research to use standard methodology to examine universal constructs; transcultural literature has noted the limitations presented by imported scales. One major limitation is that some constructs tend to be culture-specific, e.g., guanxi (translated loosely as "connections" or "social relationships") and renching (literally: "relationship orientation") are important motivating forces in many East Asian cultures, but do not have a precise parallel in the West [7].

Existing family functioning scales are based on limited frameworks and may generate inconsistent evaluations of family functioning for different members of a single family [8]. The Survey of Family Environment (SFE), a newly developed family functioning scale [9], has resolved these issues and has become well established. This study therefore aimed to examine the reliability and validity of a Chinese version of the SFE.

\section{Method}

\subsection{Measures}

\subsubsection{SFE}

The SFE, based on the Family Environment Assessment Model (FEAM) and Hohashi's Concentric Sphere Family Environment Theory (CSFET) [10], examines families' functioning, i.e., satisfaction score (SS), and needs for family support, i.e., needs score (NS). The CSFET is a middle-range family nursing theory that holistically addresses the family's current environment and aims to improve family well-being. It was originally written in Japanese (SFE-J) and developed with Japanese families ( $n$ of families $=1990)$ [9]. The SFE-J has demonstrated good reliability and validity in research using paired partners in child-rearing families. Internal consistency was measured using Cronbach's alpha, the obtained values for which indicated high consistency (SS: 0.94, NS: 0.93). Temporal stability over a two-week interval was supported by high and significant intraclass correlation coefficients (SS: 0.92, NS: 0.90) ( $n$ of families $=1990)$ [9]. The SFE-J's total score was significantly correlated with scores on the Japanese version of the Feetham Family Functioning Survey (FFFS) [11], indicating acceptable concurrent validity $\left(\mathrm{r}_{\mathrm{s}}=-0.41, \mathrm{p}<0.0001\right)$. Construct validity was supported by confirmatory factor analysis (CFA) that used a five-factor structure to examine the SFE-J's correlation with CSFET (GFI [goodness of fit index], AGFI [adjusted goodness of fit index], NFI [normed fit index], and RMR [root mean square residual] $=0.982,0.989,0.976$, and 
0.030, respectively). The SFE-J's family functioning scores showed no significant difference between paired partners [9].

The instructions were given to evaluate the satisfaction level for each item based not upon "perception by individual family members" but by "perception as a family unit," as shown in Figure 1, and is therefore able to evaluate family functioning without discrepancies between family members, including cases when responses are given by only a single family member [9].

\subsubsection{Scoring}

The SFE is essentially a norm-referenced instrument [12] and a self-administered questionnaire consisting of 30 items structured into the following domains: suprasystem, macrosystem, microsystem, family internal environment system, and chronosystem (Table 1). Participants assign a satisfaction score (SS) and an importance score (IS) to each item using a 5 -point Likert scale $(1=$ dissatisfied/unimportant, $5=$ satisfied/important; see Figure 1). The needs score (NS) indicates the family's need for support. It is obtained for each item by cross calculating the IS against a 6 - SS (with scores ranging from 1 to 25 points). The average value of the response from the couple was treated as the family's score, and if a response could only be obtained from a single family member, then this response was treated as the family's score.

In the items related to work, children, and home care, boxes for checking inapplicable (INAP) were provided. When respondents checked INAP, those items were excluded from the scoring. As the number of items to which the respondents replied varied, the overall scores or scores broken down by domains were calculated using the item average [9].

\subsection{The Chinese Translation Process}

We obtained permission to develop a Chinese version of SFE from the SFE-J's develop-

Please be sure to read the explanatory cover sheet. We want to stress that responses concerning the level of satisfaction and importance should involve your entire family, and not only yourself.

In the case of four of the 30 items, if the other choices do not apply to you, please circle INAP that appears on the far right margin. When you select INAP, it is not necessary to reply in accordance with satisfaction level and importance level.

\footnotetext{
1 Family's going on leisure outings together

2 Family members who hold jobs work with enthusiasm (If no family members are currently employed, circle INAP only.)
}
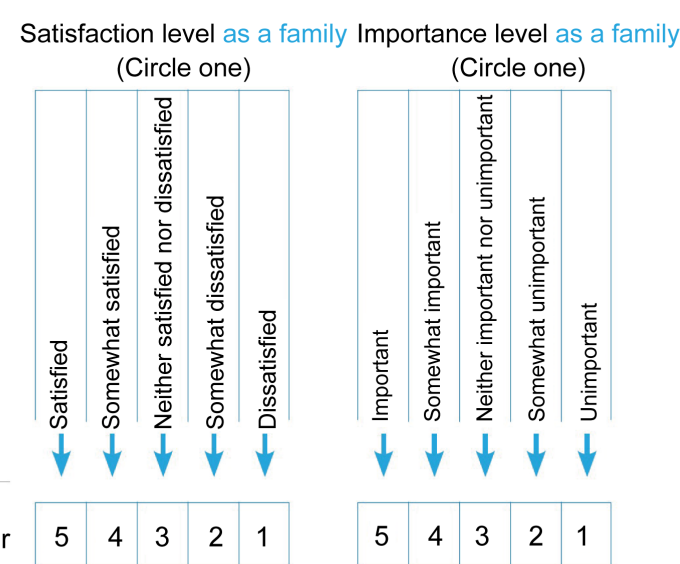

\begin{tabular}{l|l|l|l|l|}
\hline 5 & 4 & 3 & 2 & 1
\end{tabular}

\begin{tabular}{l|l|l|l|l|}
5 & 4 & 3 & 2 & 1
\end{tabular}

Figure 1. A sample of the questionnaire items. 
Table 1. The Survey of Family Environment (SFE).

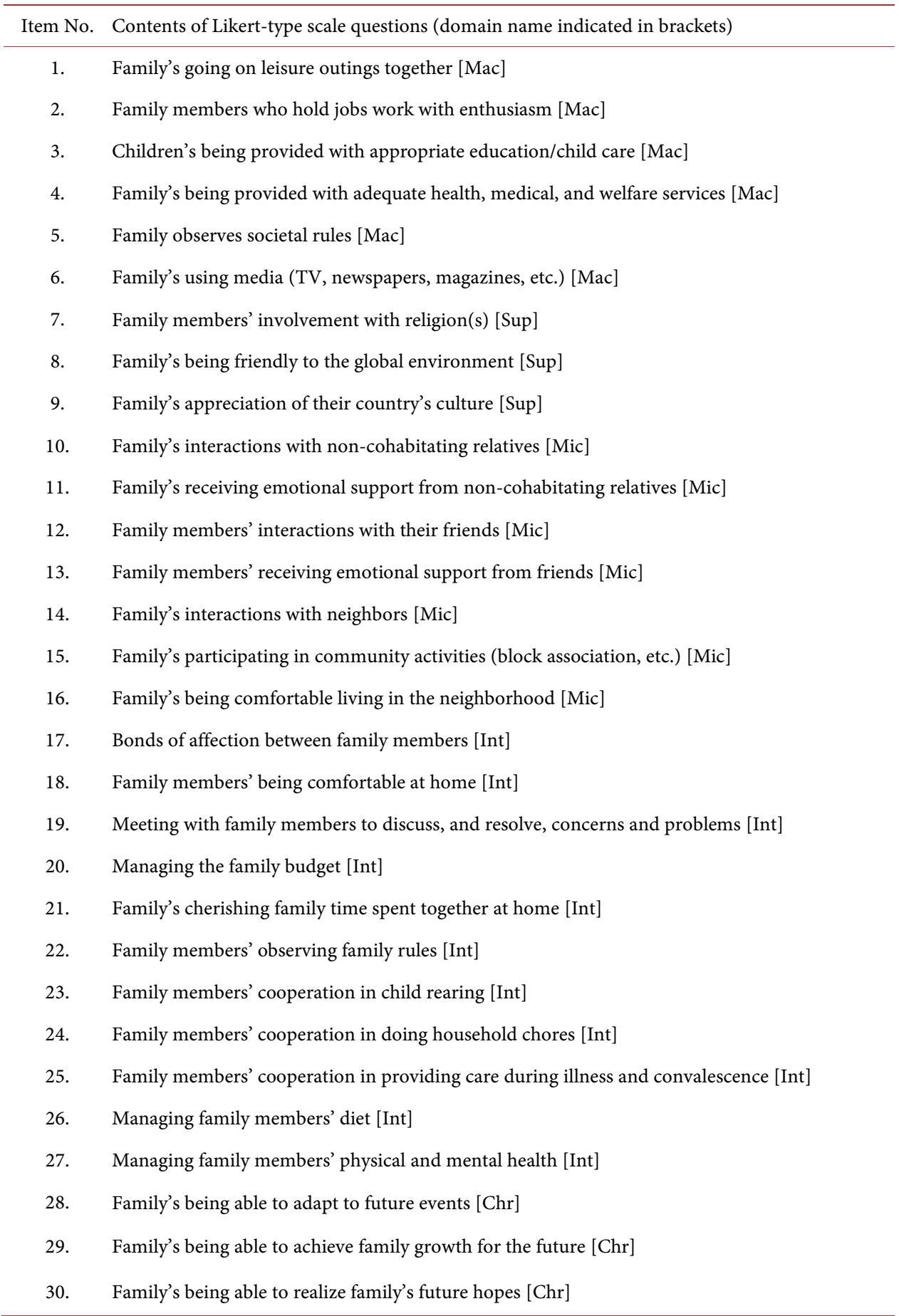

Note: Sup = suprasystem; $\mathrm{Mac}=$ macrosystem; $\mathrm{Mic}=$ microsystem; Int = family internal environment system; $\mathrm{Chr}=$ chronosystem. Items number 2, 3, 23, and 25 contain a box where INAP (Inapplicable) may be selected.

ers. The SFE-J was translated into Chinese by two expert translators. Then a Chinese expert translator and a Chinese researcher compiled a complete translation based on the former translation with attention to accuracy and conciseness. An expert Japanese 
translator who is familiar with the Chinese language then confirmed this translation's accuracy and a draft of the SFE-C was composed. A nursing researcher in Hong Kong, a Chinese graduate nursing student living in Japan, and two Japanese nursing researchers discussed this draft and revised its expressions to enable the widest possible applicability in countries and regions where Chinese is spoken.

\subsection{Participants}

Following the SFE-J, this study examined child-rearing families. A list of kindergartens in Hong Kong (available on the Education Bureau of Hong Kong SAR's website: http://www.chsc.hk/kindergarten/?lang=e) was used to select the examined settings. Six kindergartens in Hong Kong's three main districts (i.e., Hong Kong Island, Kowloon, and the New Territories) agreed to distribute our questionnaires to parents; 1340 families received our questionnaires. According to Hatcher [13], when conducing factor analysis, the number of participants is recommended to be over five times the number of items. As the SFE-C consists of 30 items, over 150 participants were recruited.

\subsection{Ethical Considerations}

Ethical approval for this research was obtained from the institutional review board (IRB) of the researchers' university. Participants received an explanation of the research's purpose and that participation was voluntary; all participants were assured of confidentiality by a cover letter. Participants were advised that the questionnaire was anonymous and that although the numbering of questionnaires used by husbands and wives would enable identification of paired partners, this would not enable identification of individual participants. Completion and return of the questionnaire was considered to indicate informed consent.

\subsection{Data Collection Procedures and Instruments}

Our questionnaires included a family sociodemographic sheet [14], the SFE-C, and the Chinese version of the FFFS (FFFS-C) [15]. The FFFS is based on Roberts \& Feetham's family ecological model (1982); it examines three areas of family functioning: "relationship between family and family members," "relationship between family and the subsystem," and "relationship between family and society." In this context, "subsystem" refers to the family's status as a component of society (i.e., the macroscopic system [16]). The FFFS is a 25-item self-administered questionnaire; responses use a 7-point Likert scale. Item scores are summed; higher scores indicate less sufficient family functioning [3].

Similar to the SFE, the FFFS assesses the external and internal family environments. No other well-established and widely accepted instrument comparable to the SFE is available; the FFFS-C was therefore used to examine the SFE-C's convergent validity, i.e., criterion-related validity. The FFFS-C's validity and reliability have been examined among child-rearing Japanese families [15]. Values of Cronbach's alpha for FFFS-C scores were 0.91 in an instrument development study, indicating high internal consis- 
tency. In a test-retest study examining 39 mothers, total scores' correlation coefficient over a two-week period was 0.82 , indicating high test-retest reliability. CFA and exploratory factor analysis (EFA) indicated that the FFFS-C has a five-factor structure based on the family ecological model, thereby supporting its construct validity. The FFFS was used in the study that examined the SFE-J's convergent validity; the present study therefore also used the FFFS-C.

A self-administered questionnaire examining families' demographics was produced following earlier research [8]; this questionnaire examined family composition, household income, family members' age, employment status, and educational level, and whether individual family members had any disease or illness [14].

\subsection{Operational Definitions of Basic Terms}

"Family" was defined as unit or organization composed of individuals recognized as belonging to the family by other constituent member(s) of the family. Families were therefore composed of parents, spouses (including cohabitants and common-law and de facto marriage partners), children, and others, independent of cohabitation. "Children" referred to all family members aged less than 18 years. These terms are explained in the "Completion Instructions" included on the first page of the SFE-C. "Family functioning" referred to family's cognitive activities performed by a family and its abilities to act on the family environment through individual family members' role behavior. Family functioning was measured as the family unit's perceived satisfaction with the current state of family functioning as assessed by the individual family member.

\subsection{Data Analysis}

All statistical analysis used SPSS v. 21.0 and Amos v. 21.0 for Microsoft Windows (IBM Inc.). Values of $\mathrm{p}<0.05$ were considered statistically significant.

Reliability was estimated by measuring internal consistency using Cronbach's alpha and a test-retest procedure. Internal consistency was measured regarding the SFE-C's total score and each domain's score. The test-retest procedure involved administering the SFE-C to a single group twice over a two-week interval; this permitted estimation of score stability using inter-correlation confidence (ICC). The standard interpretation of effect size was used, i.e., small $=0.10$, medium $=0.30$, large $=0.50$ [17] .

A Chinese nursing researcher, a Chinese graduate nursing student studying in Japan, and two Japanese nursing researchers examined content validity during the SFE-J's translation into Chinese. The SFE-C's convergent validity was estimated using score correlations with the FFFS-C.

CFA was used to estimate the SFE-C's construct validity by testing the SFE-J's factor structure's data fit. Data fit was measured using the chi-squared test, adjusted goodness of fit index (AGFI), comparative fit index (CFI), root mean square error of approximation (RMSEA), and Akaike's information criterion (AIC). A non-significant chi-square value, AGFI and CFI $>0.90$, and RMSEA $<0.05$ indicate good fit. Smaller AIC values indicate a better model. Additionally, regarding cultural considerations, EFA was per- 
formed to examine conceptual differences between the SFE-C and SFE-J.

\section{Results}

The researchers obtained 479 paired data sets $(n$ of families $=479)$. The response rate in terms of number of families was 30.5\% (479/1340). Among these, husbands' mean SS score was $3.89(S D=0.52)$ and wives' mean SS score was $3.85(S D=0.50)$. The Wilcoxon signed-rank test regarded each paired husband and wife's SS as a test pair. SS scores did not differ significantly within couples; couples' mean SS score was therefore used as a family functioning score in this study, in accordance with the SFE-J [9] [18].

\subsection{Family Sociodemographics}

Valid responses were obtained from 519 families (the response rate was 38.7\% [519/ 1340]); Table 2 shows families' sociodemographic data. Among these families, 33 responded to the temporal stability assessment (33 husbands and 33 wives) and 155 responded to the convergent validity assessment ( 74 husbands and 81 wives).

\subsection{Reliability}

\subsubsection{Internal Consistency}

Table 3 shows values of Cronbach's alpha, which was used to estimate reliability via internal consistency; values were SS $=0.92$, IS $=0.89$, and NS $=0.89$ for the overall

Table 2. Descriptive statistics for the sample of child-rearing families.

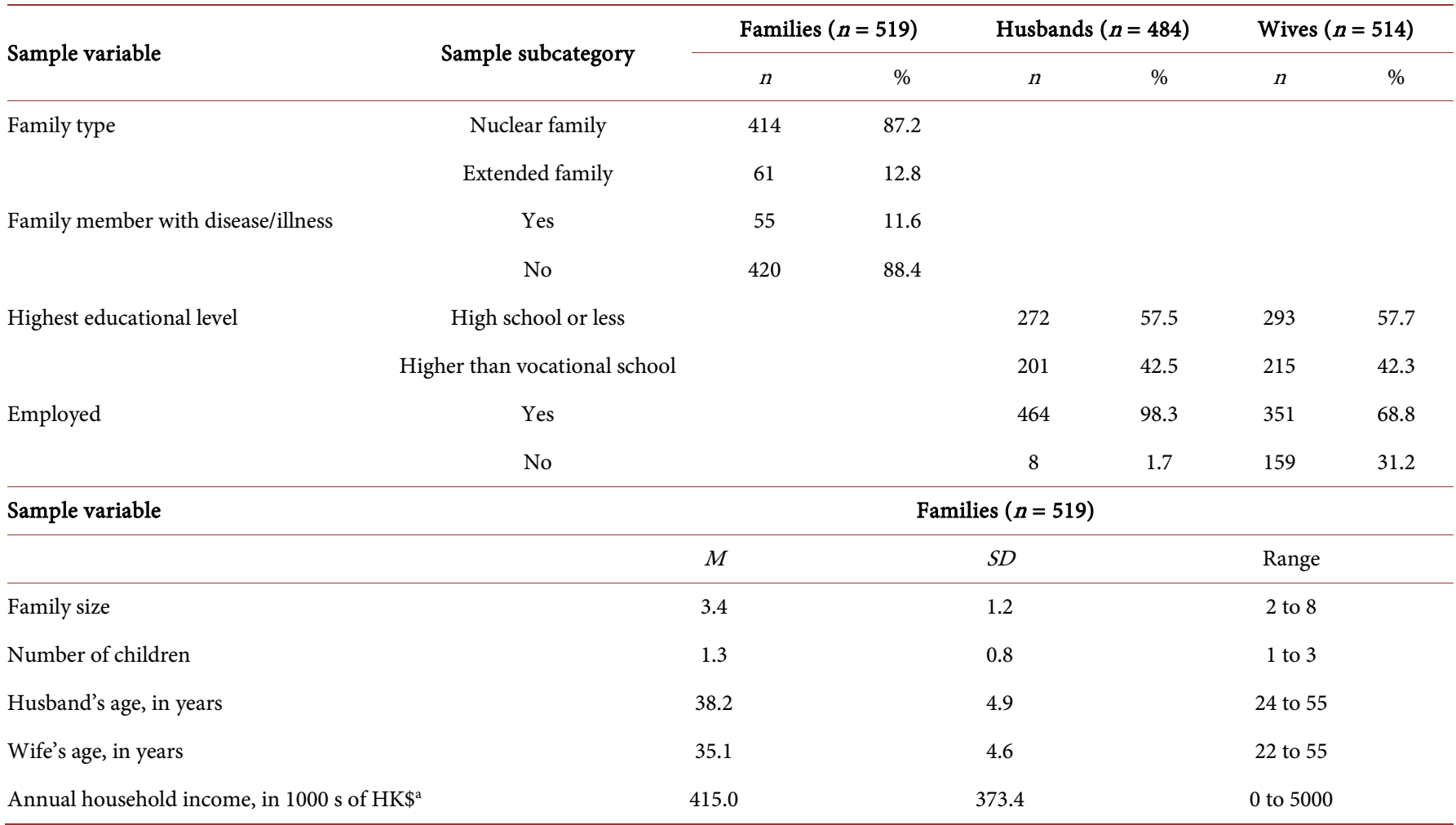

Note: Several cases were excluded from analysis due to incomplete data. a. US $\$ 1=7.8 \mathrm{HK}$ (exchange rate at time of study). 
Table 3. Cronbach's alpha of family's scores for the overall scale and each subscale.

\begin{tabular}{|c|c|c|}
\hline Scale & Score & Cronbach's alpha \\
\hline \multirow[t]{6}{*}{ SS (Satisfaction Score) } & Sup (3 items) & $0.60[0.77]^{\mathrm{a}}$ \\
\hline & Mac (6 items) & 0.65 \\
\hline & Mic (7 items) & 0.82 \\
\hline & Int (11 items) & 0.83 \\
\hline & Chr (3 items) & 0.87 \\
\hline & Overall (30 items) & 0.92 \\
\hline \multirow[t]{6}{*}{ IS (Importance Score) } & Sup (3 items) & $0.41[0.72]^{\mathrm{a}}$ \\
\hline & Mac (6 items) & 0.57 \\
\hline & Mic (7 items) & 0.80 \\
\hline & Int (11 items) & 0.81 \\
\hline & Chr (3 items) & 0.84 \\
\hline & Overall (30 items) & 0.89 \\
\hline \multirow[t]{6}{*}{ NS (Needs Score) } & Sup (3 items) & $0.51[0.69]^{\mathrm{a}}$ \\
\hline & Mac (6 items) & 0.60 \\
\hline & Mic (7 items) & 0.72 \\
\hline & Int (11 items) & 0.80 \\
\hline & Chr (3 items) & 0.86 \\
\hline & Overall (30 items) & 0.89 \\
\hline
\end{tabular}

Note: $n$ of families $=519$. Sup $=$ suprasystem; Mac $=$ macrosystem; Mic $=$ microsystem; Int $=$ family internal environment system; $\mathrm{Chr}=$ chronosystem. a. If item number 7 (the item related to religion[s] in the suprasystem) was deleted, Chronbach's alpha is indicated in brackets.

score and $0.41-0.87$ in each subscale. Cronbach's alpha was low regarding suprasystem; however, omission of item 7 (which examined religion[s]) following previous research raised this subscale's Cronbach's alpha to $>0.69$.

\subsubsection{Temporal Stability}

Table 4 shows results from the two-week test-retest. ICC values between the first and second test were as follows: overall SS score $=0.93$, overall IS score $=0.78$, and overall NS score $=0.94$.

\subsection{Validity}

\subsubsection{Convergent Validity}

The correlation coefficient between FFFS-C and SFE-C scores was -0.31 .

\subsubsection{Construct Validity}

CFA was performed to examine the SFE-C's factor structure and to test if the SFE-C has five factors to accord with the CSFET' five systems (Table 1). The values of GFI, AGFI, NFI, and RMR were $0.87,0.85,0.82$, and 0.03 , respectively; these statistics indi- 
Table 4. Test-retest correlation coefficients for family's scores over a two-week period.

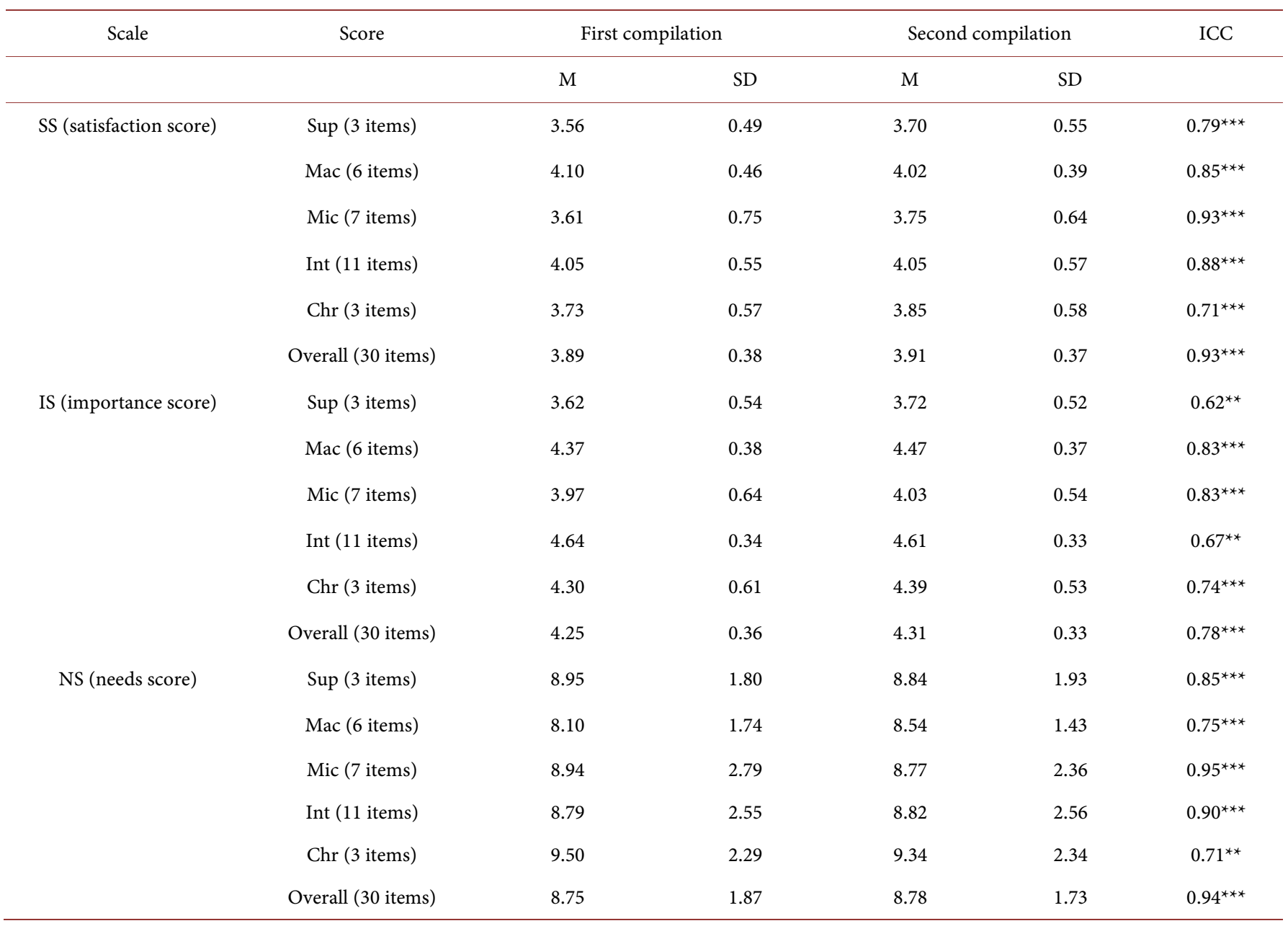

Note: Sup = suprasystem; Mac $=$ macrosystem; Mic $=$ microsystem; Int $=$ family internal environment system; Chr $=$ chronosystem; ICC $=$ intraclass correlation coefficients. The interval between first compilation and second compilation is two weeks. $n=33,{ }^{* *} \mathrm{p}<0.01,{ }^{* * *} \mathrm{p}<0.001$.

cated that the data marginally fit the model. EFA subsequently indicated that the SFE-C has seven factors (Table 5). These were as follows: "family internal environment system", "microsystem", "macrosystem", "chronosystem”, "suprasystem”, "nursing care and household chores", and "management of daily living". Two items examining childrearing and media did not belong to any factors.

\section{Discussion}

The present results indicate that the SFE-C, which is based on CSFET, is a valid and reliable means of measuring family functioning and needs in Chinese-speaking populations. Earlier research has developed family functioning scales; however, these do not adequately address the whole family [7]. Importantly, in the present research, the SFE-C obtained consistent scores between paired respondents, i.e., husbands and wives, thereby indicating that the SFE-C is able to measure Chinese-speaking families' functioning and needs including in cases where only a single family member responds. 
Table 5. Seven-factor solution of exploratory factor analysis with promax rotation.

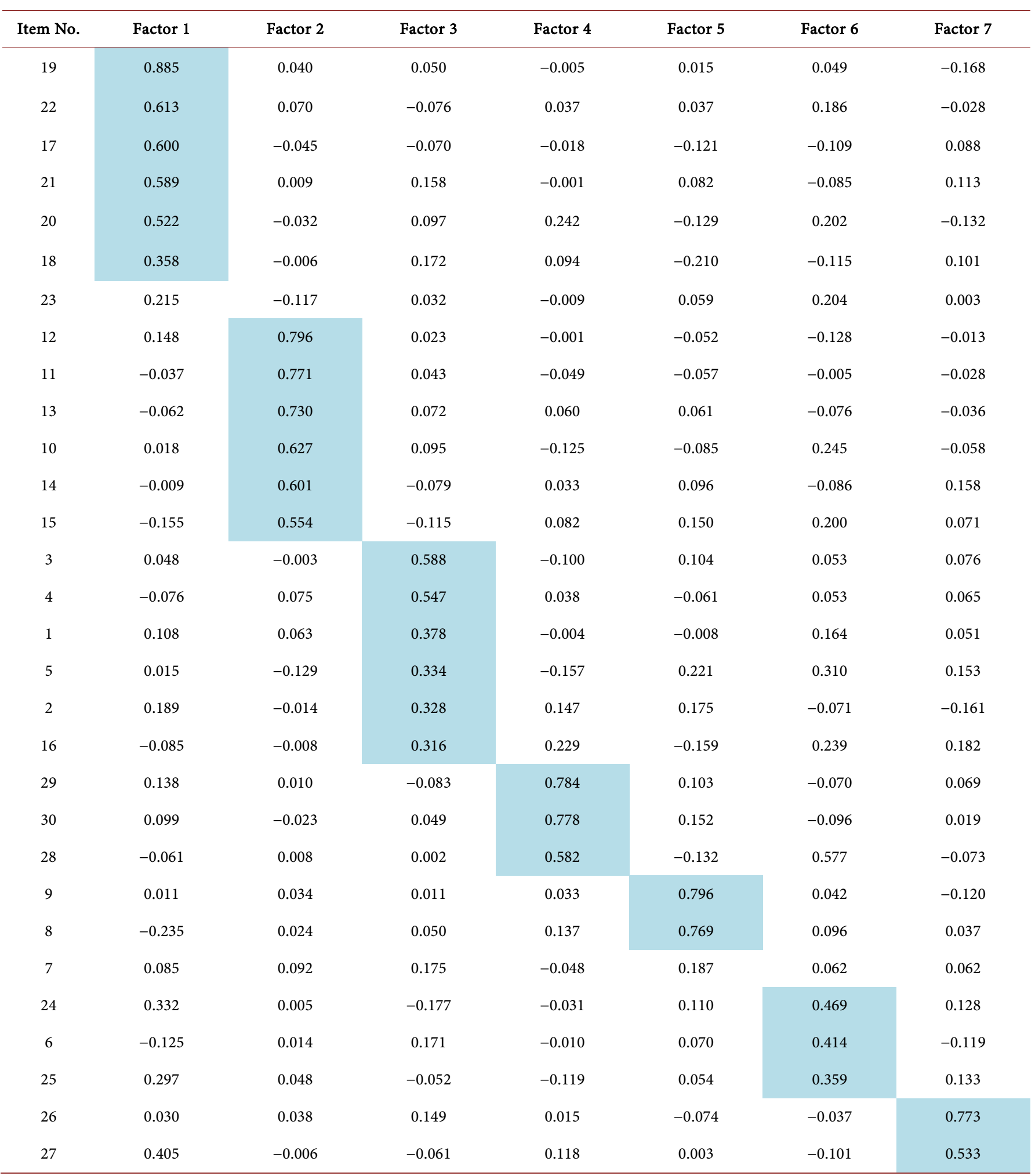

Note: $n$ of families $=519$, Extraction method: maximum likelihood, Rotation method: promax with Kaiser normalization. Factor $1=$ family internal environment system; Factor 2 = microsystem; Factor 3 = macrosystem; Factor 4 = chronosystem; Factor 5 = suprasystem; Factor $6=$ nursing care and household chores; Factor 7 = management of daily living. The items belonging to the respective factors are highlighted in blue. 


\subsection{The SFE-C's Reliability}

The Cronbach's alpha values for SFE-C's overall scores were SS $=0.92$, IS and NS $=$ 0.89 ; these values indicate that the SFE-C is highly reliable. Nonetheless, regarding individual subsystems' results, values of Cronbach's alpha tended to be smaller in the suprasystem and macrosystem than in other systems. This trend was also observed during the SFE-J's development. Several individual items in each of these systems appeared to substantially contribute to these low Cronbach's alpha values. Adhering to the practice of the SFE-J's developers, excluding an item examining religion[s] in the suprasystem elevated this system's Cronbach's alpha value to $>0.69$. The SFE-J was developed in Japan, and its developers mentioned that the item examining religion tended to reduce the scale's internal consistency as religion, in general, does not play a dominant role in Japanese family life. Although religion in Hong Kong and Japan have different contexts in terms of its effect on family functioning, these differences may not be of major significance, as compared, for example to ethnic groups in Malaysia, where preceding research found that religion less strongly affects living satisfaction among ethnic Chinese (who might practice one or several religions, including Buddhism, Taoism and Christianity) as opposed to ethnic Malay people, who tend to be mostly Muslim [19]. The CSFET proposes that religion is important related to family function [8]; therefore, the item examining religion was retained in the SFE-C.

\subsection{The SFE-C's Validity}

We used CFA to test the hypothesis that the SFE-C would have the same factor structure as the CSFET. The results indicated marginal data fit. Construct validity was therefore supported using the CSFET; however, as the fit was marginal, we conducted EFA to further examine the SFE-C's factor structure. EFA indicated that the SFE-C incorporates seven factors, of which the first through fifth correspond to the CSFET's factor structure. We named the sixth factor "nursing care and household chores" and the seventh factor "management of daily living." These factors may have been excluded from the internal family environment in the present research because families in Hong Kong often entrust domestic tasks such as household chores and caring for family members to others, for example by engaging domestic helpers or by dining out [20]. An item examining child-rearing did not belong to any factor due to low factor load; however, as the highest factor load on this item came from "family internal environment system" and the second-highest came from "nursing care and household chores," this item was considered acceptable. Overall and among Chinese families, these results suggest that "nursing care and household chores", "management of daily living", and child-rearing are situated at the borderline between the internal and external family environment systems. The CSFET proposes that an interface exists between family systems and that each system interact with the others [10]. Considering the background of the families that participated in this study, the present EFA results may reflect interaction between families' internal environment and microsystems. This further suggests that the SFE's factor structure may be able to capture interaction between family sys- 
tems when the SFE is used to examine families with different sociocultural backgrounds. Additionally, factor analysis may indicate the location of SFE items' examined parameters in target families' subsystems. That is, researchers may be able to determine items' relative functional and structural distance from family system units [10]. An item examining media (item No. 7), did not belong to any factor as its factor loading was less than 0.2 . The reason for the low figure is not fully understood and further research will be needed.

The SFE-C's development makes possible transcultural study using the SFE-C and SFE-J in combination. The English version of the SFE is under development, and future research developing the SFE in other language versions is also planned.

\section{Limitations}

Although precautions were taken with the vocabulary of the SFE-C to enable usage throughout Chinese-speaking regions, the areas where Chinese is utilized are extensive, and because this study applies only to Hong Kong, the issue remains concerning the study's reliability and validity in all Chinese-speaking regions. Furthermore, this study applies to child-rearing families, and it is supposed that future investigation will be needed to ascertain as to whether it will also apply to other families, including families in which members have a particular illness.

\section{Conclusion}

This study supported the reliability and validity of the SFE-C as a measure of family functioning and needs in Chinese-speaking families.

\section{Acknowledgements}

This project was supported in part by a Grant-in-Aid for Scientific Research (B) from the Japan Society for the Promotion of Science (JSPS) in Japan (Grant No. 15H05084).

\section{Declaration of Conflicting Interests}

The authors declare that they have no competing interests.

\section{References}

[1] Braun, V.F., Hyndman, K. and Foster, C. (2010) Family Nursing for Undergraduate Nursing Students: The Brandon University Family Case Model Approach. Journal of Family Nursing, 16, 161-176. http://dx.doi.org/10.1177/1074840710366565

[2] Smilkstein, G. (1978) The Family APGAR: A Proposal for a Family Function Test and Its Use by Physicians. Journal of Family Practice, 6, 1231-1239.

[3] Roberts, C.S. and Feetham, S.L. (1982) Assessing Family Functioning across Three Areas of Relationships. Nursing Research, 31, 231-235. http://dx.doi.org/10.1097/00006199-198207000-00011

[4] Epstein, N.B., Baldwin, L.M. and Bishop, D.S. (1983) The McMaster Family Assessment Device. Journal of Marital and Family Therapy, 9, 171-180. http://dx.doi.org/10.1111/j.1752-0606.1983.tb01497.x 
[5] Skinner, H.A., Steinhauer, P.D. and Santa-Barbara, J. (1983) The Family Assessment Measure. Canadian Journal of Community Mental Health, 2, 91-103.

http://dx.doi.org/10.7870/cjcmh-1983-0018

[6] Beavers, W.R., Hampson, R.B. and Hulgus, Y.F. (1985) Commentary: The Beavers Systems Approach to Family Assessment. Family Process, 24, 398-405.

http://dx.doi.org/10.1111/j.1545-5300.1985.00398.x

[7] Lee, P.H., Stewart, S.M., Lun, V.M.C., Bond, M.H., Yu, X. and Lam, T.H. (2012) Validating the Concord Index as a Measure of Family Relationships in China. Journal of Family Psychology, 26, 906-915. http://dx.doi.org/10.1037/a0029994

[8] Honda, J. and Hohashi, N. (2015) Discrepancies between Couples' Perceptions of Family Functioning in Child-Rearing Japanese Families. Nursing \& Health Sciences, 17, 57-63. http://dx.doi.org/10.1111/nhs.12148

[9] Hohashi, N. and Honda, J. (2012) Development and Testing of the Survey of Family Environment (SFE): A Novel Instrument to Measure Family Functioning and Needs for Family Support. Journal of Nursing Measurement, 20, 212-229. http://dx.doi.org/10.1891/1061-3749.20.3.212

[10] Hohashi, N. and Honda, J. (2011) Development of the Concentric Sphere Family Environment Model and Companion Tools for Culturally Congruent Family Assessment. Journal of Transcultural Nursing, 22, 350-361. http://dx.doi.org/10.1177/1043659611414200

[11] Hohashi, N., Maeda, M. and Sugishita, C. (2000) Development of the Japanese-Language Feetham Family Functioning Survey (FFFS) and Evaluation of Its Effectiveness. Japanese Journal of Research in Family Nursing, 6, 2-10.

[12] Waltz, C.F., Strickland, O.L. and Lenz, E.R. (2010) Measurement in Nursing and Health Research. Springer, New York.

[13] Hatcher, L. (1994) A Step-by-Step Approach to Using the SAS System for Factor Analysis and Structural Equation Modeling. SAS Institute, Inc., Cary.

[14] Hohashi, N. and Honda, J. (2016) Assessment Guide for Japanese Version of the SFE Family Sociodemographics Module (SFE/FSD-J). Editex, Tokyo.

[15] Hohashi, N., Honda, J. and Kong, S.K. (2008) Validity and Reliability of the Chinese Version of the Feetham Family Functioning Survey (FFFS). Journal of Family Nursing, 14, 201 223. http://dx.doi.org/10.1177/1074840708315967

[16] Bronfenbrenner, U. (1979) The Ecology of Human Development: Experiments by Nature and Design. Harvard University Press, Cambridge.

[17] Cohen, J. (1992) A Power Primer. Psychological Bulletin, 112, 155-159. http://dx.doi.org/10.1037/0033-2909.112.1.155

[18] Hohashi, N. and Honda, J. (2016) Assessment Guide for Japanese Version of the Survey of Family Environment (SFE-J). Editex, Tokyo.

[19] Rakrachakarn, V., Moschis, G.P., Ong, F.S. and Shannon, R. (2015) Materialism and Life Satisfaction: The Role of Religion. Journal of Religion and Health, 54, 413-426. http://dx.doi.org/10.1007/s10943-013-9794-y

[20] Honda, J. and Hohashi, N. (2007) An Ethnographic Study on Family Functions of ChildRearing Households in Hong Kong: With a Focus on the Implementation of Family Functions. Japanese Journal of Research in Family Nursing, 13, 45-52. 